\title{
Parkinson's disease: chameleons and mimics
}

\author{
Khalid Ali, ${ }^{1}$ Huw R Morris ${ }^{2,3,4}$
}

\begin{abstract}
'Department of Neurology, Royal Gwent Hospital, Newport, Gwent, UK

${ }^{2}$ Department of Clinical Neuroscience, UCL Institute of Neurology, London, UK ${ }^{3}$ Department of Neurology, Royal Free Hospital, London, UK ${ }^{4}$ Neurology, National Hospital for Neurology, London, UK
\end{abstract}

\section{Correspondence to} Professor Huw R Morris, Department of Clinical Neuroscience, UCL Institute of Neurology, Upper 3rd Floor, Royal Free Hospital, Rowland Hill Street, London NW3 2PF, UK; h.morris@ucl.ac.uk

Accepted 18 August 2014 Published Online First 24 September 2014

\section{CrossMark}

To cite: Ali K, Morris HR. Pract Neurol 2015;15:14-25.

\section{ABSTRACT}

Parkinson's disease (PD) is a common neurodegenerative condition that usually presents with symptoms related to asymmetric bradykinesia, resting tremor, rigidity and postural instability. Making the correct diagnosis can be challenging as many conditions_-including tremor, gait and atypical parkinsonian disorders — can mimic PD. PD can present with unanticipated motor and non-motor symptoms, and so can masquerade as a number of rheumatological, neurological, sleep and mood disorders. Careful clinical assessment, informed by well-validated diagnostic criteria, is important in the initial diagnostic formulation. In uncertain or ambiguous cases, follow-up with monitoring of the treatment response usually gives the correct diagnosis, as validated in postmortem follow-up studies. 'Premotor' PD—a range of non-motor symptoms, particularly sleep disorders and constipation, which can occur up to 20 years before PD motor onset-is common. The presence of non-motor features in early disease sometimes supports the diagnosis of PD. Here we give an overview of the diagnosis of PD and its most important chameleons and mimics, and review the recent advances in structural and functional imaging in parkinsonism.

\section{INTRODUCTION}

Parkinson's disease (PD) is a common clinical condition affecting approximately $1 \%$ of the population older than 65 years. ${ }^{12}$ Many lay people and non-specialists inevitably associate PD with tremor. However, tremor is a presenting feature in only half of all patients with PD, although 90\%$100 \%$ of patients with PD have tremor at some stage in their disease course. ${ }^{3-6}$ There are other common causes of tremor - as well as conditions that cause slowness or a change in gait and posture-that can present as PD mimics. ${ }^{7}$ Our concept of PD has changed from a pure movement disorder to a clinicopathological entity with protean features, including changes in mood, sleep, behaviour, cognition and autonomic function. PD can cause symptoms that lead to referrals to psychiatry, general medicine, care of the elderly, orthopaedic and rheumatology clinics, before arriving at the correct primary underlying diagnosis. A definite diagnosis of PD can only be made at postmortem by identifying degeneration of the substantia nigra pars compacta and the presence of Lewy bodies-insoluble cytoplasmic inclusions containing aggregated alphasynuclein. Although early diagnosis is occasionally difficult, by the time of death at least $90 \%$ of postmortem-proven patients with PD have a correct clinical diagnosis. ${ }^{8}$

\section{TYPICAL PD AND THE CLINICAL EXAMINATION}

The distinctive pathological features of PD have led to robust and well-validated clinical diagnostic criteria. In 1988, Gibb and Lees developed the Queen Square Brain Bank (QSBB) criteria for PD, based on careful clinical observation combined with clinicopathological correlation. ${ }^{9}$ In 1992, Hughes and colleagues showed that only $76 \%$ of patients diagnosed as having PD by UK neurologists fulfilled the neuropathological diagnostic criteria for the disease. Retrospective application of the QSBB clinical criteria improved the diagnostic accuracy to $82 \%$. Multiple system atrophy, progressive supranuclear palsy, Alzheimer's disease, Alzheimer-like pathology and vascular parkinsonism comprised most of the alternative diagnoses. ${ }^{10}$ The same group's subsequent study in 2001 showed that the clinical diagnosis accuracy had improved to $90 \% .^{11}$ The QSBB criteria remain a benchmark for PD diagnosis, and careful application and appreciation of the criteria will prevent most pitfalls for the clinician. However, it is important to appreciate that many patients with PD may not fulfil the QSBB criteria for PD at presentation (box 1). Expert clinicians are reportedly more 
Box 1 UK Queen Square Brain Bank Criteria for the diagnosis of Parkinson's disease ${ }^{9}$

Step 1

Diagnosis of a parkinsonian syndrome

Bradykinesia (slow to initiate voluntary movement with progressively reduced speed and amplitude of repetitive actions) and at least one of the following:

- Muscular rigidity

- Rest tremor $(4-6 \mathrm{~Hz})$

- Postural instability unrelated to primary visual, cerebellar, vestibular or proprioceptive dysfunction

Step 2

Exclusion criteria of Parkinson's disease

History of:

- Repeated strokes with stepwise progression

- Repeated head injury

- Antipsychotics or dopamine-depleting drugs

- Definite encephalitis and/or oculogyric crisis on no drug treatment

- More than one affected relative (Note: this exclusion criteria is usually ignored)

- Sustained remission

- Negative response to large doses of levodopa (if malabsorption excluded)

- Strictly unilateral features after 3 years

- Other neurological features: supranuclear gaze palsy, cerebellar signs, early severe autonomic involvement, Babinski's sign, early severe dementia with disturbance of language, memory or praxis

- Exposure to known neurotoxin

- Presence of cerebral tumour or communicating hydrocephalus on neuroimaging

Step 3

Supportive criteria for Parkinson's disease

Three or more required for the diagnosis of definite Parkinson's disease

- Unilateral onset

- Rest tremor present

- Progressive disorder

- Persistent asymmetry affecting the side of onset most

- Excellent response to levodopa $(70 \%-100 \%)$

- Severe levodopa-induced chorea

- Levodopa response for more than 5 years

- Clinical course of over 10 years

Box courtesy of Professor Andrew Lees

accurate than the QSBB criteria at diagnosing PD, and this presumably relates to the integration of a number of sometimes subtle historical and examination findings. ${ }^{8}{ }^{11}$ Parkinson's syndrome (stage 1 of the QSBB criteria) is defined by bradykinesia and one additional feature of tremor, rigidity or postural instability. Bradykinesia can be appreciated as a general slowness and paucity of movement, particularly in a decrease in the normal spontaneous fidgeting and gestural movements that occur during the interview. Parkinsonian bradykinesia is specifically elicited by examining repeated movements, usually finger taps, hand grips, pronation-supination movements, toe taps and heel stamps, following the scheme of the Movement Disorders Society unified PD rating scale (MDS-UPDRS). In PD, there is a specific form of bradykinesia involving progressive loss of the speed and amplitude of repetitive movements and there may be arrests in movements and 're-setting'. There is also a perceptible slowness of initiation and subtle weakness. The MDS-UPDRS scheme mandates that these movements are assessed over 10 repetitions. ${ }^{12}$ Many other factors can affect the ability to carry out repeated movements, including arthritis, pyramidal deficits, dyspraxia, depression, obsessional slowness, cerebellar disease, dystonia and other cognitive deficits. These concurrent comorbidities may make identification of true bradykinesia difficult in some patients, particularly the elderly. It is important that parkinsonian bradykinesia is not 'over-diagnosed' and these PD-bradykinesia mimics are a potential pitfall for the unwary. Bradykinesia can be difficult to assess in patients with marked postural tremor. If there is uncertainty as to whether there is genuine parkinsonian bradykinesia, we refer to the abnormality of movement as 'clumsiness' or of 'slowness of movement without decrement', pending further diagnostic information. A history of fatigable movement ('I can start brushing my teeth normally but then the movements fade out'), the presence of generalised bradykinesia and the ability to assess multiple movements (ie, arms and legs) as outlined above can help in assessing ambiguous cases.

It is important to appreciate that fulfilling stage 1 criteria is not equivalent to fulfilling the QSBB diagnostic criteria for PD as there should be no exclusion criteria, and three supportive criteria should be present (box 1). The supportive criteria include the presence of a typical $4-6 \mathrm{~Hz}$ resting tremor. Patients without a typical resting tremor are unlikely to fulfil the QSBB criteria for PD at presentation, as six of the other features relate to disease course and treatment response. Resting tremor can be assessed in clinic by asking the patient to relax in a chair with arms supported and asking them to carry out a distraction task, such as counting backwards or lying on the couch in a relaxed position. In PD, the tremor normally stops on starting a movement but may re-emerge with sustained posture (re-emergent tremor). PD tremor can affect the lips, chin and legs, while head and neck tremor points towards other disorders. ${ }^{3}$ PD is an asymmetrical disease, and asymmetry at onset and through the disease course are both important supportive criteria. Additional features that have emerged since the publication of the QSBB criteria-and that may help to support a diagnosis of PD early in the disease course -include anosmia, prodromal constipation and rapid eye movement (REM) sleep behaviour disorder. 
Observing the clinical course and progression of a patient with suspected PD is one of the most important aspects of making an accurate diagnosis. ${ }^{13} \mathrm{PD}$ is progressive, and disability related to cognition and gait usually occurs more than 5 years after disease onset. The response to drug treatment is an important part of the assessment. Patients with PD usually respond well to levodopa, but $20 \%-40 \%$ of patients develop levodopa-induced dyskinesia within 5 years, although in most patients this is not disabling. ${ }^{14} \mathrm{In}$ $\mathrm{PD}$, particularly in patients with akinetic-rigid dominant presentations, there is usually a marked $(>70 \%)$ beneficial response to levodopa treatment. ${ }^{9}$

\section{PD CHAMELEONS}

Failure to recognise PD can relate to non-neurological or atypical presentations of motor and/or non-motor symptoms (box 2). PD causes stiffness and slowness, which also occur in a plethora of rheumatological conditions, leading to missed and delayed diagnoses. Many patients also have a prodromal non-motor syndrome related to underlying Lewy body pathology. Identifying patients with prodromal PD or 'Parkinson's at risk' subjects is now a major research endeavour, ${ }^{15}$ with a view to starting early diseasemodifying therapy. From a clinical standpoint, we recommend regular follow-up of patients with, for example, REM sleep behaviour disorder, as they have an increased risk of developing PD, multiple system atrophy and dementia with Lewy bodies. ${ }^{16-18}$

These motor and non-motor symptoms can lead to referral to various specialties, including orthopaedics, spinal surgery, urology, psychiatry, care of the elderly and general practice. ${ }^{16} \mathrm{PD}$ can also be misdiagnosed as any of its mimics mentioned below. ${ }^{16}$

\section{Non-motor symptoms}

It is well established that patients with PD can have non-motor symptoms before developing the motor

\section{Box 2 Parkinson's disease chameleons}

Non-motor related
Sensory features and pain
Sleep-related disorders
Autonomic features (urinary urgency)
Mood disturbance (depression and anxiety)
Motor related
Stroke
Spinal degenerative disease
Frozen shoulder
Asymmetric motor performance, for example, difficulty in
swimming in a straight line or skiing
Exercise-induced dystonia
Note: Bold entries are common presentations

features of PD. One study showed that 21\% of autopsy-proven patients with PD presented initially with exclusively non-motor symptoms. ${ }^{8}$ Patients who presented with non-motor symptoms had a delayed diagnosis of PD at 1.6 years compared with 1.0 years for patients with motor presentations. ${ }^{8}$

Sensory symptoms: Pain is the most frequent nonmotor presentation of PD, accounting for up to $15 \%$ of all PD presentations and is, therefore, the most common PD chameleon. ${ }^{8}$ Pain in PD most likely relates to the motor-affected side. Many patients with PD have been previously diagnosed to have osteoarthritis, degenerative spinal disease and frozen shoulder due to both non-motor (pain) and motor symptoms. Some patients may be referred to orthopaedic surgeons, spinal surgeons or rheumatologists and may have unnecessary procedures, ${ }^{8} 16$ although some patients also develop musculoskeletal disorders secondary to parkinsonian bradykinesia and stiffness. Hyposmia is a common non-motor symptom in PD, occurring in $80 \%-90 \%$ of patients and preceding the motor symptoms by up to 40 years. In a large prospective study, impaired olfaction predated the diagnosis of PD by 4 years. ${ }^{19}$ However, it is rarely an isolated presenting symptom. ${ }^{17}{ }^{18}$ Hyposmia can occur in asymptomatic first-degree relatives of patients with familial PD. ${ }^{20}$

REM sleep behaviour disorder is usually reported by the patient's partner and can precede the diagnosis of PD by many years. The witness may report vivid dreams of a violent nature with the patient screaming, yelling and punching during sleep. ${ }^{21}$ Several studies report that $80 \%$ of patients with the idiopathic form who present to sleep disorders clinics eventually develop a neurodegenerative disorder-most commonly PD—but sometimes include other synuclein disorders such as multiple system atrophy and dementia with Lewy bodies. ${ }^{22-24}$ However, REM sleep behaviour disorder is rare at the presentation of PD, with one study showing a prevalence of $<4 \%$ at initial assessment. It is particularly associated with visuospatial and cognitive dysfunction; the development of REM sleep behaviour disorder predicts the development of daytime hallucinations. ${ }^{8} 2526$

Autonomic features: Autonomic features are common in PD; some, particularly constipation, can precede the movement disorder. Patients may report increased difficulty passing faeces and incomplete bowel emptying; this may prompt multiple gastroenterological investigations before the underlying diagnosis of PD becomes apparent. Constipation can predate the motor features of PD by up to 12 years. ${ }^{27}$ A small proportion (4\%) of all patients with PD present with urinary urgency. ${ }^{8}$ This can lead to misdiagnosis of benign prostatic hypertrophy. ${ }^{16}$ In one large prospective study of early PD, hypersalivation and dribbling occurred in 55\% of patients and urinary urgency was reported in $46 \%$ of patients within the 
first year of diagnosis. ${ }^{28}$ In another prospective study of patients with idiopathic REM sleep behaviour disorder, there was autonomic dysfunction 5 years before the diagnosis of $\mathrm{PD}^{29}$ Other autonomic features include erectile dysfunction, dizziness and hyperhidrosis, although these features usually do not predate the onset of PD. ${ }^{8} 29$ Disturbed thermoregulation and sweating are common prodromal features of PD or at presentation, although it is relatively unusual for disturbed sweating to lead to a medical consultation.

Neuropsychiatric features: Several case-control and prospective studies have shown a high prevalence of depression and anxiety in early PD and in the premotor phase of the disease. Depression occurs up to 10 years before PD diagnosis; ${ }^{28}$ 30-32 studies suggest that $15 \%$ of patients with PD develop depression in the early stages of the disease or before diagnosis. Depression as a presenting feature of PD occurs in $2.5 \%$ of the cases and so this can be a PD chameleon. ${ }^{8}$ Another study showed increased incidence of PD in depressed adult patients. ${ }^{33}$ This is particularly common in elderly patients where the onset of severe depression in a patient previously unaffected by psychiatric disease can indicate emergent PD or dementia with Lewy bodies. Other non-motor symptoms include cognitive impairment, anxiety and lethargy. ${ }^{8}$

\section{Motor symptoms}

The asymmetry of PD means that some patients presenting with an apparent sudden onset illness may be erroneously diagnosed as having had a stroke. ${ }^{8} 16$ Atypical presentations can relate to both asymmetrical stiffness and sensory symptoms. Many patients describe their symptoms in terms of shoulder/arm pain and stiffness, and may develop secondary rheumatological problems, such as frozen shoulder $^{34}$; this may lead to inappropriate or unnecessary treatment. Further unusual motor presentations include those related to asymmetrical motor performance in complex tasks and sports, including swimming and skiing. Exercise-induced dystonia occurs in about $20 \%$ of patients with early-onset PD and may be prominent in some patients with parkin disease. ${ }^{35}$

\section{PD MIMICS}

The most important PD mimics include tremor disorders, drug-induced parkinsonism, vascular parkinsonism and Parkinson's-plus conditions (box 3 and table 1). Patients with these diseases are often misdiagnosed as having PD. Evidence for the existence of PD mimics comes from functional imaging studies of patients who were initially diagnosed to have PD and from pathological follow-up of clinically diagnosed PD cases. These studies relate to both early-stage and late-stage diagnostic accuracy. The difficulties caused by PD mimics have recently been highlighted by the identification of patients recruited to PD treatment trials, passing apparently stringent diagnostic
Box 3 Selected Parkinson's disease mimics

Tremor

- Dystonic tremor

- Isolated rest tremor

- Indeterminate tremor

- Essential tremor

- Fragile $\mathrm{X}$ tremor-ataxia syndrome

Primary gait disorders

- Progressive supranuclear palsy

- Vascular parkinsonism/frontal gait disorder

- Normal pressure hydrocephalus

Atypical parkinsonian disorders

- Multiple system atrophy

- Corticobasal degeneration

- Dementia with Lewy bodies

Drug-induced parkinsonism

- Neuroleptics

- Dopamine blocking antiemetics

- Sodium valproate

Depression

Early-onset and genetic parkinsonian disorders

- Wilson's disease

- Juvenile Huntington's disease

- Spinocerebellar ataxias

- Fronto-temporal dementia with parkinsonism

- Pallido-pyramidal syndromes/neurodegeneration with brain iron accumulation

assessment for PD, who had normal functional dopaminergic imaging (ie, scans without evidence of dopaminergic deficits (SWEDDS)) and therefore, probably do not have PD. ${ }^{36-38}$ Movement disorders patients with SWEDDS represent a heterogeneous group of patients with a PD-like presentation. Possible explanations are dystonic and essential tremor, depression, vascular parkinsonism, psychogenic disease and dopa-responsive dystonia. ${ }^{38-40}$ The SWEDDS studies suggest that red flags for PD mimics include lack of true parkinsonian bradykinesia, occurrence of major dystonia and the clinical characteristics of the tremor. $^{38} 39$ We will briefly outline the clinical features of the PD mimics.

\section{Tremor disorders}

Patients with tremor commonly present to general neurology and movement disorder clinics: often the patient prompts the referral with concerns about possible PD. Most patients can be reassured after clinical assessment. Most patients with significant non-PD tremor who are referred to movement disorders clinics have dystonic tremor; however, there is some controversy on this point and diagnostic practices differ between the UK and other countries. ${ }^{41}$ Patients with both dystonic tremor and essential tremor frequently have action tremor and postural tremor rather than a 
Table 1 Clinical pointers and radiological features in parkinsonian syndromes

\begin{tabular}{|c|c|c|}
\hline & Clinical pointers & Radiological features \\
\hline $\begin{array}{l}\text { Multiple system } \\
\text { atrophy }\end{array}$ & $\begin{array}{l}\text { May be indistinguishable from PD in early stages } \\
\text { Jerky finger tremor related to mini-polymyoclonus } \\
\text { 'Strangulated' dysarthria } \\
\text { Axial/cranio-cervical levodopa-induced dyskinesia }\end{array}$ & $\begin{array}{l}\text { Cerebellar atrophy } \\
\text { T2 high signal in degenerating pontocerebellar fibres } \\
\text { leading to 'hot-cross bun' sign (figure 2) } \\
\text { T2 low signal in putamen with rim of increased signal } \\
\text { on lateral edge }\end{array}$ \\
\hline $\begin{array}{l}\text { Progressive } \\
\text { supranuclear palsy }\end{array}$ & $\begin{array}{l}\text { Erect posture with good step size at presentation } \\
\text { - Frequent falls and injuries early in the disease course } \\
\text { - Slowed saccadic eye movements may be subtle in early disease }\end{array}$ & $\begin{array}{l}\text { Midbrain atrophy (with 'hummingbird' sign on sagittal } \\
\text { brainstem images, figure 3) } \\
\text { - 3rd ventricle dilatation }\end{array}$ \\
\hline $\begin{array}{l}\text { Corticobasal } \\
\text { degeneration }\end{array}$ & $\begin{array}{l}\text { - Markedly asymmetrical rigid/akinetic/apraxic limb with relatively } \\
\text { normal contralateral limb in early disease }\end{array}$ & - Asymmetric fronto-parietal atrophy on MRI \\
\hline Essential tremor & $\begin{array}{l}\text { High-frequency tremor } \\
\text { The tremor is postural and kinetic, and improves with rest } \\
\text { Absent PD non-motor features } \\
\text { Head and neck tremor } \\
\text { - May have a long and benign course }\end{array}$ & - DaTscan is normal \\
\hline Dystonic tremor & $\begin{array}{l}\text { Thumb extension tremor } \\
\text { Jerky tremor with flurries of tremor } \\
\text { - May be task-specific or task-exacerbated }\end{array}$ & - DaTscan is normal \\
\hline Vascular parkinsonism & $\begin{array}{l}\text { Presents as gait disorder } \\
\text { 'Lower body' parkinsonism with mild or absent upper body } \\
\text { parkinsonism }\end{array}$ & $\begin{array}{l}\text { Neuroimaging shows variable degrees of small-vessel } \\
\text { ischaemic changes }\end{array}$ \\
\hline
\end{tabular}

PD, Parkinson's disease.

rest tremor; some have a rest component, leading to diagnostic difficulties. Tremor assessment should include examination of patients in a relaxed position with arms supported, looking for rest tremor in sustained posture and during action. Rest tremor may only become apparent during other distraction tasks, such as counting backwards, walking or contralateral finger tapping. ${ }^{42}{ }^{43}$ Action tremor may develop during finger-to-nose movement. We routinely examine patients' handwriting, both in drawing Archimedes' spirals and in writing a sentence ${ }^{44}$ (figure 1). Dystonic tremor may be particularly severe when pouring water from cup to cup. The clinical classification of tremor disorders-particularly those likely to be confused with PD—may be difficult, but clinical pointers are very important. Important features of non-PD tremor include a marked writing tremor and an action tremor more prominent than rest tremor.

Dystonic tremor: The features of dystonic tremor include thumb extension tremor, jerkiness of tremor with flurries and task or position specificity. Patients do not have true parkinsonian bradykinesia and respond poorly to levodopa. Although concurrent dystonia may help, some patients with PD, particularly in early-onset disease, have prominent dystonia - with an extended thumb or 'dinner-fork' posturing - so this cannot be a definitive distinguishing feature. Dystonic tremor may be a major cause of disease in patients with SWEDDS. In patients with dystonic and essential tremor, dopamine-transporter scan $\left({ }^{123}\right.$ I-FP-CIT SPECT scan (DaTscan)) is normal, indicating that there is no presynaptic dopaminergic deficit. ${ }^{39} 404546$

Monosymptomatic rest tremor: Some patients present with a rest tremor that has the same clinical characteristics as PD tremor but without bradykinesia or without other features of PD. If this persists for more than two years, then this can be called 'monosymptomatic rest tremor'. Functional imaging in these patients may show dopaminergic denervation, suggesting that this condition is often a clinically restricted form of PD. These patients should not be diagnosed as having PD unless they develop bradykinesia, which in some patients never occurs or begins only after a long time delay. ${ }^{43} 47$

Indeterminate tremor disorder: According to the MDS Tremor Investigation Group classification, patients with indeterminate tremor satisfy the criteria for definite or probable essential tremor, but have either recognisable neurological disorders such as parkinsonism or have neurological signs of unclear significance, making the diagnosis of essential tremor doubtful. ${ }^{47}$

Essential tremor: This is probably the most common movement disorder, although many patients do not seek medical attention. Classical essential tremor is both action and postural, predominantly affecting the hands and is usually symmetrical. Essential tremor may be very mild. Classical essential tremor is relatively unlikely to be confused with PD, although essential tremor may be a potential cause of tremor in patients with SWEDDS. ${ }^{38}$ There may be a positive family history and beneficial response to alcohol. ${ }^{48-51}$

Fragile X tremor-ataxia syndrome (FXTAS) causes a progressive intention and postural tremor, with parkinsonism and cerebellar features. MR scan of brain shows cortical and subcortical atrophy with symmetrical lesions in the periventricular white matter and characteristically increased T2 signal in the middle cerebellar peduncle. FXTAS is caused by a pre- 
Dystonic tremor

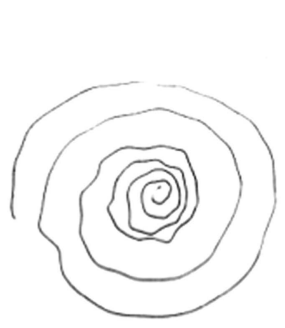

Left

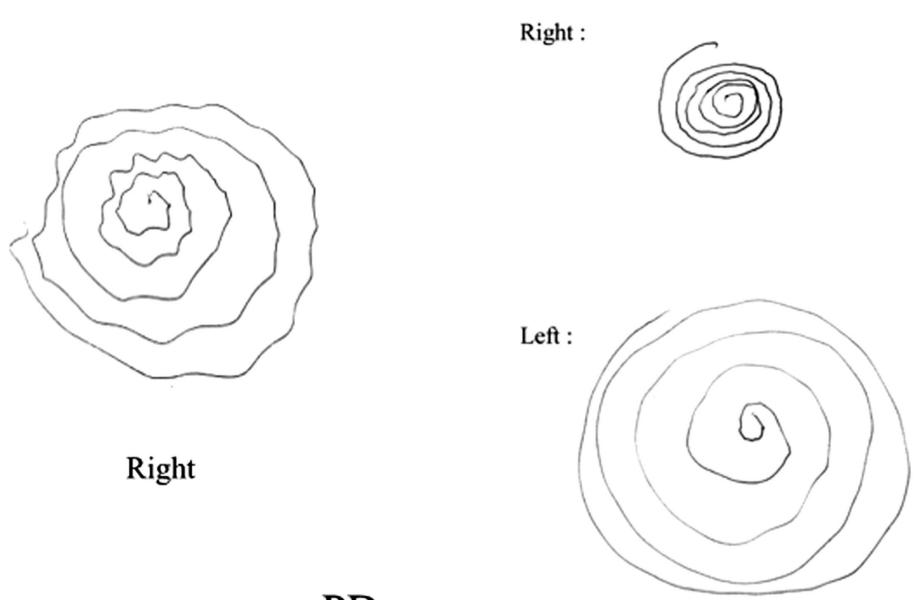

PD

PD an uncontrolled descent when sitting. The typical form of the disease (progressive supranuclear palsyRichardson's syndrome) is distinctive, involving a symmetric akinetic-rigid syndrome, axial rigidity, supranuclear gaze palsy, continuous frontalis overactivity, reduced blink rate, retrocollis and pseudobulbar palsy. The bradykinesia involves small-amplitude finger taps without decrement. ${ }^{54}$ In the early phases of the disease, there may be slowing rather than restriction of saccadic eye movements. Some forms of progressive supranuclear palsy-diagnosed pathologically-more closely resemble PD in the early disease stages (progressive supranuclear palsy-parkinsonism) and show an initial moderate response to levodopa. ${ }^{16} 5556$

Vascular parkinsonism/frontal gait disorder: Vascular parkinsonism/diffuse cerebral small vessel disease is the most common cause of a frontal gait disorder. The diagnosis is based on the clinical features and the exclusion of normal pressure hydrocephalus, structural frontal lesions and conditions such as progressive supranuclear palsy. Frontal gait disorders ('gait apraxia') usually involve a short-stepped gait, without upper body parkinsonism, usually with an erect posture and normal or exaggerated arm swing. Patients may have asymmetrical difficulty in positioning the foot or leg when walking. Some patients have

Figure 1 Writing in Dystonic tremor and Parkinson's disease: Dystonic tremor - no micrographia and asymmetric tremor; Parkinson's disease - asymmetric progressive micrographia.

mutation expansion (55-200 repeats) of the CGG trinucleotide repeat in the fragile $\mathrm{X}$ mental retardation-1 (FMR1) gene. Although FXTAS can be mistaken for $\mathrm{PD}$, it is more likely to be mistaken for multiple system atrophy-cerebellar subtype or cerebellar ataxia. $^{52} 53$

\section{Gait disorders}

PD rarely presents primarily with a gait disorder and falls, though this can occur, particularly in the elderly. Presentation with a gait disorder suggests an alternative diagnosis. Close attention to posture, neck position and movement, arm position and arm swing is very helpful in diagnosing PD and its mimics. Patients with PD usually have a flexed trunk and neck posture with reduced arm swing and flexed elbows and a reduced step size. Normal or erratic step size, extended neck postures and normal or exaggerated arm swing are red flags, suggesting alternative diagnoses.

Progressive supranuclear palsy: This tauopathy is a primary disorder of gait and balance, as well as an atypical parkinsonian disorder. The gait disorder is characterised by a normal step size and erect, sometimes hyperextended posture, often with a lurching gait. In the early disease stages, patients with progressive supranuclear palsy have difficulty in standing and 
gait freezing when standing or turning. The management is focused mainly on treating vascular risk factors and providing supportive therapy for mobility and falls prevention. High-dose levodopa helps some patients with vascular parkinsonism, although this has not been formally evaluated in randomised controlled trials. As with PD, patients with vascular parkinsonism can respond well to auditory and visual cues and verbal instructions. ${ }^{57-59}$

Normal pressure hydrocephalus causes a similar clinical syndrome to vascular parkinsonism. Patients present with progressive cognitive decline, subcortical dementia, urinary incontinence and a frontal gait disorder. Brain imaging shows ventriculomegaly, disproportionate to the degree of cerebral atrophy. The diagnosis is confirmed by cerebrospinal fluid (CSF) removal or by dynamic CSF studies, such as a lumbar infusion test or a lumbar drain. CSF removal may improve gait, cognition or both. Some patients may benefit from a permanent CSF diversion procedure. Around $70 \%$ of these who undergo CSF diversion procedures show some initial gait improvement, but this often subsequently declines. Only one-third have sustained improvement at 3 years; the cognitive and urinary function generally has a worse long-term outcome than does gait. ${ }^{60-62}$

\section{Atypical parkinsonian disorders}

Multiple system atrophy: This synucleinopathy can be difficult to distinguish from PD, particularly in the early disease stages. Patients with multiple system atrophy often have asymmetrical parkinsonian bradykinesia with a good response to levodopa and may develop levodopa-induced dyskinesia. They may develop a flexed trunk and neck posture, identical to PD. Although autonomic features and gait disturbance are typical of multiple system atrophy, they can occur in PD. However, the relationship between these symptoms and disease onset is important. In multiple system atrophy, urinary incontinence, postural hypotension and gait disturbance are usually present at diagnosis or in the first few years after presentation, whereas in PD these occur later in the disease course (although constipation is often an early feature of both PD and multiple system atrophy). Useful additional clues to the diagnosis of multiple system atrophy include stretch-sensitive and action myoclonus affecting the fingers (mini-poly-myoclonus) and a predominantly cranio-cervical levodopa-induced dyskinesia often with dystonic facial movement and a 'strangulated' dysarthria. ${ }^{16} 6364$

Corticobasal degeneration: This tauopathy presents with an asymmetrical akinetic-rigid syndrome (like PD). Loss of arm swing and unilateral stiffness often lead to an erroneous diagnosis of PD. There is often marked pain together with dystonia, apraxia, myoclonus and cortical sensory loss. Corticobasal degeneration does not respond well to levodopa treatment. At presentation, positional or postural limb instability, dyspraxia and myoclonus are important distinguishing features. ${ }^{65} 66$

Dementia with Lewy bodies: Dementia with Lewy bodies, PD and PD-dementia share common pathological and clinical features, and relate to a continuum of brainstem, limbic and neocortical Lewy body pathology. By definition, dementia with Lewy bodies involves major cognitive symptoms within the first year of onset, usually with parkinsonism. To some extent, the separation of these conditions is arbitrary and clearly some patients with dementia with Lewy bodies closely mimic those with PD. Prominent visual hallucinations and fluctuations in attention and awareness are important features in the diagnosis of dementia with Lewy bodies. ${ }^{67} 68$

\section{Drug-induced parkinsonism}

Various medications can cause parkinsonian symptoms and signs, including dopamine-depleting agents (such as tetrabenazine), dopamine antagonists (such as the antiemetics prochlorperazine and metoclopramide) and antipsychotics (fluphenazine, haloperidol and the depot preparations). Sodium valproate has recently been described as causing parkinsonism. High-dose cinnarizine and flunarizine can cause parkinsonism. Adulterated medicines bought on the internet containing kava-kava (Piper methysticum) and reserpine can cause severe parkinsonism. ${ }^{2}$ Illegal psychostimulant drugs containing ephedrine with manganese have caused severe drug-resistant parkinsonism in Eastern European countries, related to cerebral manganese toxicity. ${ }^{69}$ Sometimes these drugs may unmask underlying PD. The parkinsonian effects of these drugs can take up to a year to resolve after cessation of the medication. The DaTscan is normal in drug-induced parkinsonism, primarily due to blockade of D2 dopamine receptors. ${ }^{2} 4569-71$

\section{Depression/psychiatric disease}

Slowing of movement and motor retardation, related to severe depression, can lead to the erroneous identification of bradykinesia and parkinsonism. Depression has been highlighted as one of the possible causes of SWEDDS and obsessional slowness can result from obsessive-compulsive disorder. ${ }^{39} 40$

\section{EARLY-ONSET AND GENETIC PARKINSONISM}

Parkinsonism can occur in a wide variety of early-onset neurological and neurodegenerative diseases. We describe some selected conditions that can lead to diagnostic confusion below.

\section{Monogenetic PD}

PD can be a genetic/familial neurodegenerative disease. Genetic investigations may be worthwhile in patients who present before the age of 40 years or who have more than one affected relative. Familial PD 
can be autosomal dominant, for example, due to coding mutations (PARK1) or duplications/triplications (PARK4) in the alpha-synuclein gene or due to the common LRRK2 G2019S mutation. ${ }^{72}$ In general terms, patients with autosomal-dominant PD are indistinguishable from patients with sporadic $\mathrm{PD},{ }^{73}$ although some mutations, particularly the alphasynuclein E46K mutation and triplication, can present with early-onset disease and marked early dementia. $^{74-77}$ Mutations in parkin (PARK2), DJ1 (PARK7) and PINK1 (PARK6) occur in patients with early-onset disease. ${ }^{78}$ Patients with mutations in these genes have been reported to have a long disease course, with prominent dystonia, levodopa-induced dyskinesia and neuropsychiatric features without dementia, although some studies have suggested that these features relate to age at onset rather than the genetic aetiology. ${ }^{78-80}$

Wilson's disease: This is a rare autosomal-recessive disorder caused by autosomal recessive mutations in the ATP7B gene, leading to copper deposition in the liver, the basal ganglia and other organs. It would be unusual for Wilson's disease to mimic PD directly, as age at onset, concurrent dysarthria, dystonia, proximal tremor and psychiatric features usually suggest the alternative diagnosis. Nevertheless, clinicians need a high degree of suspicion in younger patients with movement disorders involving parkinsonism; this should include a careful search for Kayser-Fleischer rings with slit-lamp examination and measurement of serum caeruloplasmin. It is important to consider Wilson's disease in young people presenting with any movement disorder and/or dysarthria. ${ }^{45} 8182$

Huntington's disease: Juvenile Huntington's disease classically presents with parkinsonism; important clues are a positive family history and a concurrent fronto-behavioural syndrome. ${ }^{83}$

Spinocerebellar atrophy (SCA) types 2 and 3: SCA-2 and 3 have been described as presenting with a presentation typical of Lewy body PD with asymmetric parkinsonism and a good response to levodopa, particularly in non-Caucasian patients. ${ }^{84}$

Fronto-temporal dementia with parkinsonism: The major forms of fronto-temporal dementia relate to underlying tau, TDP-43 or FUS gene pathology, and all may present with parkinsonism. Fronto-temporal dementia with parkinsonism can be genetic, with mutations in the MAPT, GRN or C9ORF72 genes. A prominent frontal behavioural syndrome and positive family history are important clues as to the underlying diagnosis. FTDP-17 due to mutations in MAPT can present with both $\mathrm{PD}$ and progressive supranuclear palsy-like syndromes. ${ }^{85-87}$

Neurodegeneration with brain iron accumulation: This represents a group of disorders with early-onset progressive parkinsonism-dystonia, associated with iron deposition in the basal ganglia and characterised by low signal on T2-MRI. Some of these conditions may initially respond well to levodopa and lead to initial diagnostic confusion with PD. However, the appearance of atypical features such as early bulbar involvement, spasticity, pyramidal signs, dementia and supranuclear gaze palsy together with characteristic brain imaging appearances point to the underlying diagnosis. Pantothenate kinase-associated neurodegeneration accounts for $50 \%$ of neurodegeneration with brain iron accumulation. This is an autosomal-recessive condition due to a mutation of the PANK2 gene. MR brain imaging characteristically shows the 'eye of the tiger' on T2-weighted imaging with low signal in the pallidum surrounding a high-signal focus. Neuroferritinopathy is an autosomal-dominant condition with high penetrance due to mutation of the ferritin light chain gene (FTL1). This is a neurodegenerative condition characterised by progressive stereotyped dystonic movements with orofacial dyskinesia and normal cognition. The MR scan of brain shows cystic degeneration of the basal ganglia and the 'eye of the tiger' sign may also occur in this condition. Other neurodegeneration with brain iron accumulation syndromes include acaeruloplasminaemia due to mutation of the CP gene, infantile neuroaxonal dystrophy, PLA2G6-associated neurodegeneration, FA2H, C19orf12, ATP13A2 and other conditions. ${ }^{88-92}$

\section{IMAGING BIOMARKERS IN PARKINSONISM}

Although applying the QSBB criteria gives high sensitivity and specificity in detecting established patients with PD, applying this in the early stages of the disease is more difficult when the clinical picture might not fulfil the QSBB criteria (because of lack of supportive features related to progression, response to drug treatment or because true parkinsonian bradykinesia may be difficult to assess with prominent postural tremor).

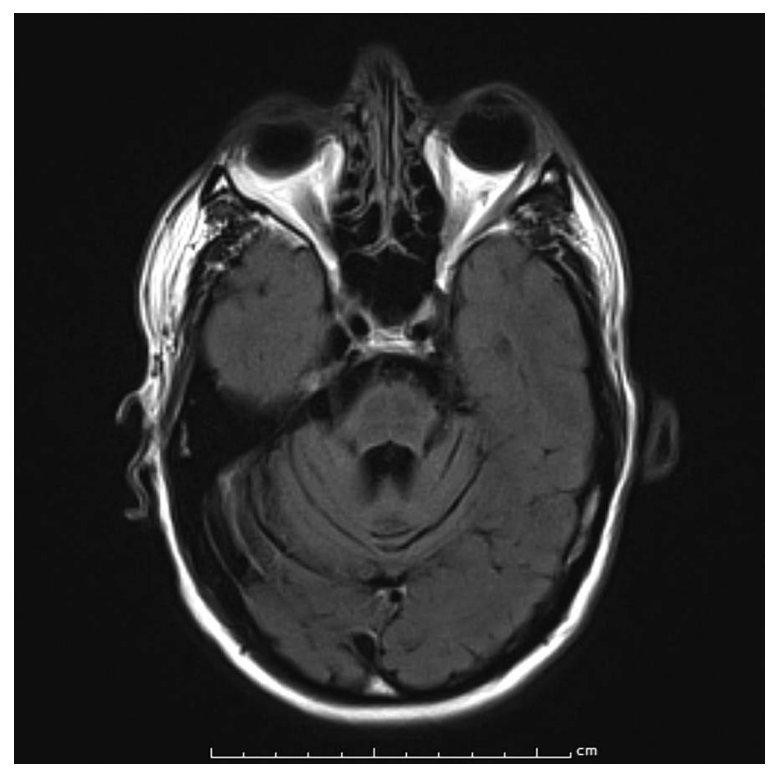

Figure 2 T1 MR scan of brain (axial) showing the 'hot-cross bun' appearance in a patient with multiple system atrophy. 


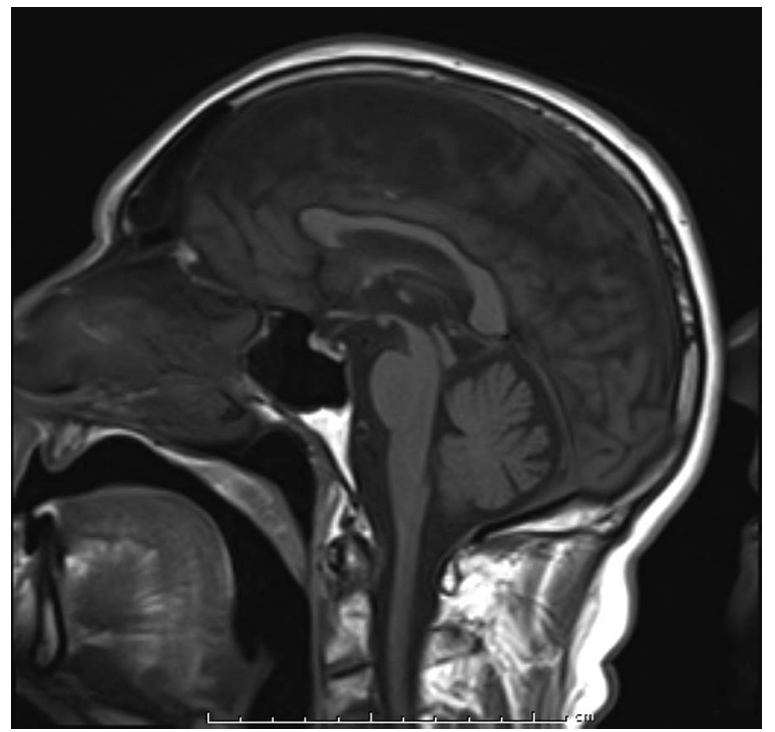

Figure 3 T1 MR scan of brain (sagittal) showing the 'hummingbird' sign in a patient with progressive supranuclear palsy.

The routine use of neuroimaging in all patients with PD is not recommended. ${ }^{93}$ The National Institute of Health and Care Excellence (NICE) in the UK recommends the use of MR scan of brain in the differential diagnosis of parkinsonian disorders but not $\mathrm{PD},{ }^{93}$ and similarly, the American Academy of Neurology (AAN) guidelines suggest that MR scan of brain can help to distinguish PD from multiple system atrophy. ${ }^{94}$ In neurological practice, MR scan of brain may be useful for patients with atypical features for PD, for example, lower body-predominant parkinsonism, strictly unilateral disease, lack of asymmetry, early urinary disturbance and early cognitive impairment. MRI particularly helps in patients with complex parkinsonian disorders - older patients may have markers of progressive supranuclear palsy, multiple system atrophy, corticobasal degeneration and vascular disease ${ }^{58}$ (table 1). MRI can detect specific abnormalities, such as pontine MRI T2 signal change (the 'hot-cross bun' sign) and reduced MRI T2 signal of the putamen in multiple system atrophy (figure 2). Midbrain, and superior cerebellar peduncle atrophy in progressive supranuclear palsy (figure 3). ${ }^{95}{ }^{96}$ In younger patients, there may be structural basal ganglia abnormalities and/or brain iron deposition, in neurodegeneration with brain iron accumulation disorders. Functional imaging of the dopamine transporter using SPECT and $\left({ }^{123} \mathrm{I}\right)$ ioflupane as a tracer (DaTscan) is well established in clinical practice as conditions such as PD, which lead to nigrostriatal dopaminergic denervation, or very rarely to loss of the dopamine transporter, lead to loss of the normal pattern on DaTscan ${ }^{97} 98$ (figure 4). The European Medicines Agency and Food and Drug Administration licences for DaTscan indicate that this tool can differentiate parkinsonian disorders from essential tremor, but that DaTscan does not differentiate PD from other parkinsonian disorders (such as progressive supranuclear palsy and multiple system atrophy). NICE and the AAN recommend a DaTscan to distinguish essential tremor from parkinsonian disorders. ${ }^{93}{ }^{94}$ DaTscan is also normal in other conditions that may mimic PD, including drug-induced parkinsonism, psychogenic tremor, some central nervous system infections, hydrocephalus, pallidal atrophies, X-linked dystoniaparkinsonism and dopa-responsive dystonia. In specialist settings where clinicians suspect these rarer PD mimics, DaTscan may be useful. ${ }^{98} 99$ Using ${ }^{123}$ iodine-meta-iodobenzyl guanidine ( $\left.{ }^{123} \mathrm{I}-\mathrm{MIBG}\right)$
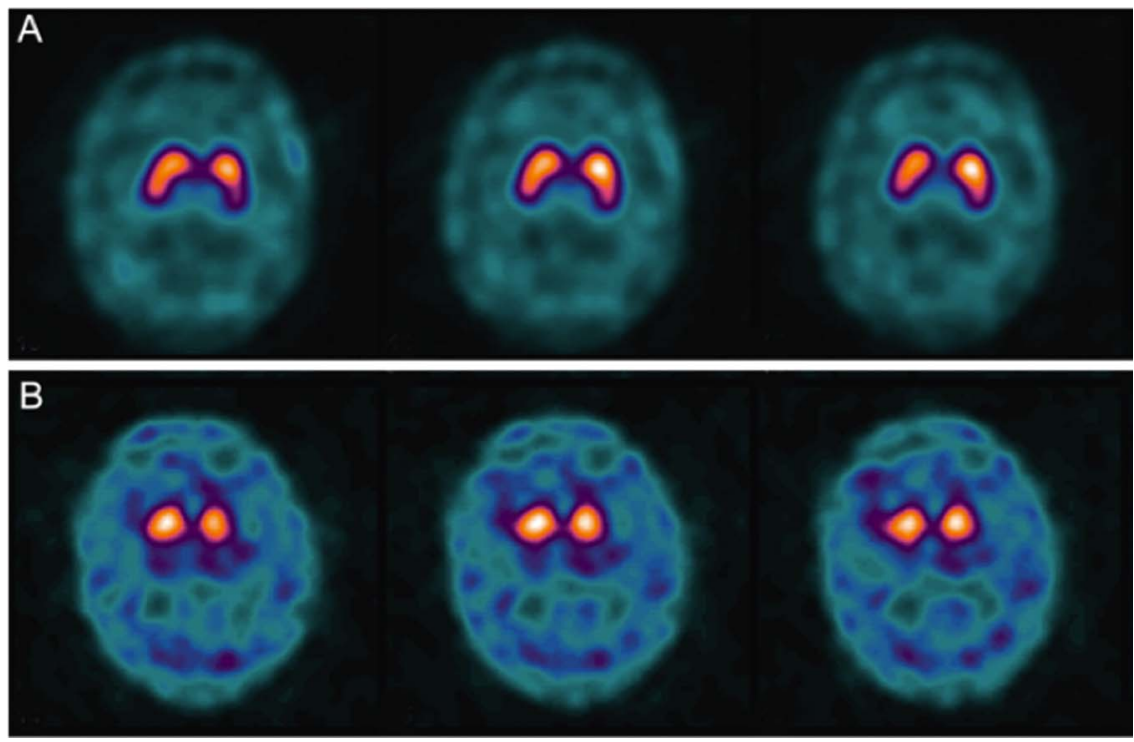

Figure 4 DaTscan: dopamine-active transporter (DaT) scans showing binding of ${ }^{123}$ I-fluoropropyl (FP-CIT) to DaT protein in the nigrostriatal nerve endings of the striatum. (A) Normal: symmetrical normal specific binding of FP-CIT in striatum. (B) Parkinson's disease: reduced specific binding of FP-CIT in the posterior striatum, particularly on the left (picture courtesy of $\mathrm{P}$ Kempster ${ }^{45}$ ). 
myocardial scintigraphy, patients with PD have significantly lower heart-to-mediastinum average count ratio than atypical parkinsonian disorders, reflecting postganglionic sympathetic cardiac denervation in PD. ${ }^{100}$ NICE and AAN guidelines do not currently recommend using this technique. ${ }^{93} 94$

Further imaging approaches are in development on a research basis. 7-T MR scanning can detect structural nigral abnormalities. ${ }^{101}$ Diffusion-tensor MRI and diffusion-weighted imaging have shown some promise in diagnosing $\mathrm{PD}$ and its mimics. $^{99}$ Transcranial sonography can detect hyperechogenicity in the midbrain of patients with PD. Although this is not specific, it may be a marker of susceptibility to PD..$^{99} 102103$

\section{CONCLUSIONS}

Over the last 20 years, the diagnosis of PD has greatly improved through the use of clinicopathological criteria, improved knowledge and understanding of non-PD movement disorders and through our improved understanding of the non-motor features of PD. It is likely that, in the coming years, imaging or fluid biomarkers will assist the clinical diagnosis of $\mathrm{PD}$, particularly in the early stages. However, at present, the most important aspects of the diagnosis of PD and the identification of PD chameleons and mimics remain the careful observation of the evolution of symptoms, signs and treatment response, and vigilance for new and atypical clinical features.

Acknowledgements We acknowledge the contribution of Dr Simon Lewis and Professor Andrew Lees, whose comments greatly strengthened this article.

Contributors KA and HRM wrote the article. KA carried out the literature search and wrote the first draft. HRM revised the article and both are responsible for the final version.

Funding HRM is supported for work on PD by Parkinson's UK (grant 8047) and the Medical Research Council UK (G0700943, G1100643).

\section{Competing interests None.}

Provenance and peer review Commissioned; externally peer reviewed. This paper was reviewed by Simon Lewis, Sydney, Australia, and Andrew Lees, London, UK.

\section{REFERENCES}

1 Wirdefeldt K, Adami H-O, Cole P, et al. Epidemiology and etiology of Parkinson's disease: a review of the evidence. Eur J Epidemiol 2011;26(Suppl 1):S1-58.

2 Lees A. Parkinson's disease. Pract Neurol 2010;10:240-6.

3 Jankovic J. Parkinson's disease: clinical features and diagnosis. J Neurol Neurosurg Psychiatry 2008;79:368-76.

4 Hughes AJ, Daniel SE, Blankson S, et al. A clinicopathologic study of 100 cases of Parkinson's disease. Arch Neurol 1993;50:140-8.

5 Martin WE, Loewenson RB, Resch JA, et al. Parkinson's disease. Clinical analysis of 100 patients. Neurology 1973;23:783-90.
6 Rajput AH, Rozdilsky B, Rajput A. Accuracy of clinical diagnosis in parkinsonism-a prospective study. Can J Neurol Sci J Can Sci Neurol 1991;18:275-8.

7 Litvan I, Bhatia KP, Burn DJ, et al. SIC Task Force appraisal of clinical diagnostic criteria for parkinsonian disorders. Mov Disord 2003;18:467-86.

8 O'Sullivan SS, Williams DR, Gallagher DA, et al. Nonmotor symptoms as presenting complaints in Parkinson's disease: a clinicopathological study. Mov Disord Off J Mov Disord Soc 2008;23:101-6.

9 Gibb WR, Lees AJ. The relevance of the Lewy body to the pathogenesis of idiopathic Parkinson's disease. J Neurol Neurosurg Psychiatry 1988;51:745-52.

10 Hughes AJ, Daniel SE, Kilford L, et al. Accuracy of clinical diagnosis of idiopathic Parkinson's disease: a clinico-pathological study of 100 cases. J Neurol Neurosurg Psychiatry 1992;55:181-4.

11 Hughes AJ, Daniel SE, Lees AJ. Improved accuracy of clinical diagnosis of Lewy body Parkinson's disease. Neurology 2001;57:1497-9.

12 Goetz CG, Tilley BC, Shaftman SR, et al. Movement Disorder Society-sponsored revision of the Unified Parkinson's Disease Rating Scale (MDS-UPDRS): scale presentation and clinimetric testing results. Mov Disord Off J Mov Disord Soc 2008;23:2129-70.

13 Caslake R, Taylor K, Scott N, et al. Age-, gender-, and socioeconomic status-specific incidence of Parkinson's disease and parkinsonism in northeast Scotland: the PINE study. Parkinsonism Relat Disord 2013;19:515-21.

14 Schrag A, Quinn N. Dyskinesias and motor fluctuations in Parkinson's disease. A community-based study. Brain J Neurol 2000;123(Pt 11):2297-305.

15 Stern MB, Siderowf A. Parkinson's at risk syndrome: can Parkinson's disease be predicted? Mov Disord Off J Mov Disord Soc 2010;25(Suppl 1):S89-93.

16 Williams DR, Lees AJ. How do patients with parkinsonism present? A clinicopathological study. Intern Med J 2009;39:7-12.

17 Massano J, Bhatia KP. Clinical approach to Parkinson's disease: features, diagnosis, and principles of management. Cold Spring Harb Perspect Med 2012;2:a008870.

18 Ziemssen T, Reichmann H. Non-motor dysfunction in Parkinson's disease. Parkinsonism Relat Disord 2007;13:323-32.

19 Ross GW, Petrovitch H, Abbott RD, et al. Association of olfactory dysfunction with risk for future Parkinson's disease. Ann Neurol 2008;63:167-73.

20 Siderowf A, Jennings D, Connolly J, et al. Risk factors for Parkinson's disease and impaired olfaction in relatives of patients with Parkinson's disease. Mov Disord Off J Mov Disord Soc 2007;22:2249-55.

21 Claassen DO, Josephs KA, Ahlskog JE, et al. REM sleep behavior disorder preceding other aspects of synucleinopathies by up to half a century. Neurology 2010;75:494-9.

22 Iranzo A, Tolosa E, Gelpi E, et al. Neurodegenerative disease status and post-mortem pathology in idiopathic rapid-eye-movement sleep behaviour disorder: an observational cohort study. Lancet Neurol 2013;12:443-53.

23 Schenck CH, Boeve BF, Mahowald MW. Delayed emergence of a parkinsonian disorder or dementia in $81 \%$ of older men initially diagnosed with idiopathic rapid eye movement sleep behavior disorder: a 16-year update on a previously reported series. Sleep Med 2013;14:744-8. 
24 Postuma RB, Aarsland D, Barone P, et al. Identifying prodromal Parkinson's disease: pre-motor disorders in Parkinson's disease. Mov Disord Off J Mov Disord Soc 2012;27:617-26.

25 Gallagher DA, Lees AJ, Schrag A. What are the most important nonmotor symptoms in patients with Parkinson's disease and are we missing them? Mov Disord Off J Mov Disord Soc 2010;25:2493-500.

26 Onofrj M, Thomas A, D’Andreamatteo G, et al. Incidence of $\mathrm{RBD}$ and hallucination in patients affected by Parkinson's disease: 8-year follow-up. Neurol Sci Off J Ital Neurol Soc Ital Soc Clin Neurophysiol 2002;23(Suppl 2):S91-94.

27 Abbott RD, Petrovitch H, White LR, et al. Frequency of bowel movements and the future risk of Parkinson's disease. Neurology 2001;57:456-62.

28 Duncan GW, Khoo TK, Yarnall AJ, et al. Health-related quality of life in early Parkinson's disease: the impact of nonmotor symptoms. Mov Disord Off J Mov Disord Soc 2014;29:195-202.

29 Postuma RB, Gagnon J-F, Pelletier A, et al. Prodromal autonomic symptoms and signs in Parkinson's disease and dementia with Lewy bodies. Mov Disord Off J Mov Disord Soc 2013;28:597-604.

30 Shiba M, Bower JH, Maraganore DM, et al. Anxiety disorders and depressive disorders preceding Parkinson's disease: a case-control study. Mov Disord Off J Mov Disord Soc 2000;15:669-77.

31 Leentjens AFG, Van den Akker M, Metsemakers JFM, et al. Higher incidence of depression preceding the onset of Parkinson's disease: a register study. Mov Disord Off J Mov Disord Soc 2003;18:414-18.

32 Gonera EG, van't Hof M, Berger HJ, et al. Symptoms and duration of the prodromal phase in Parkinson's disease. Mov Disord Off J Mov Disord Soc 1997;12:871-6.

33 Schuurman AG, van den Akker M, Ensinck KTJL, et al. Increased risk of Parkinson's disease after depression: a retrospective cohort study. Neurology 2002;58:1501-4.

34 Riley D, Lang AE, Blair RD, et al. Frozen shoulder and other shoulder disturbances in Parkinson's disease. J Neurol Neurosurg Psychiatry 1989;52:63-6.

35 Bozi M, Bhatia KP. Paroxysmal exercise-induced dystonia as a presenting feature of young-onset Parkinson's disease. Mov Disord Off J Mov Disord Soc 2003;18:1545-7.

36 Holloway RG, Shoulson I, Fahn S, et al. Pramipexole vs levodopa as initial treatment for Parkinson disease: a 4-year randomized controlled trial. Arch Neurol 2004;61:1044-53.

37 Whone AL, Watts RL, Stoessl AJ, et al. Slower progression of Parkinson's disease with ropinirole versus levodopa: The REAL-PET study. Ann Neurol 2003;54:93-101.

38 Fahn S; Parkinson Study Group. Does levodopa slow or hasten the rate of progression of Parkinson's disease? J Neurol 2005;252(Suppl 4):IV37-42.

39 Schneider SA, Edwards MJ, Mir P, et al. Patients with adult-onset dystonic tremor resembling parkinsonian tremor have scans without evidence of dopaminergic deficit (SWEDDs). Mov Disord Off J Mov Disord Soc 2007;22:2210-15.

40 Schwingenschuh P, Ruge D, Edwards MJ, et al. Distinguishing SWEDDs patients with asymmetric resting tremor from Parkinson's disease: a clinical and electrophysiological study. Mov Disord Off J Mov Disord Soc 2010;25:560-9.

41 Quinn NP, Schneider SA, Schwingenschuh P, et al. Tremor--some controversial aspects. Mov Disord Off J Mov Disord Soc 2011;26:18-23.
42 Raethjen J, Austermann K, Witt K, et al. Provocation of Parkinsonian tremor. Mov Disord Off J Mov Disord Soc 2008;23:1019-23.

43 Deuschl G, Papengut F, Hellriegel H. The phenomenology of parkinsonian tremor. Parkinsonism Relat Disord 2012;18 (Suppl 1):S87-89.

44 Bain PG, Findley LJ, Atchison P, et al. Assessing tremor severity. J Neurol Neurosurg Psychiatry 1993;56:868-73.

45 Alty JE, Kempster PA. A practical guide to the differential diagnosis of tremor. Postgrad Med J 2011;87:623-9.

46 Defazio G, Gigante AF, Abbruzzese G, et al. Tremor in primary adult-onset dystonia: prevalence and associated clinical features. J Neurol Neurosurg Psychiatry 2013;84:404-8.

47 Deuschl G, Bain P, Brin M. Consensus statement of the Movement Disorder Society on Tremor. Ad Hoc Scientific Committee. Mov Disord Off J Mov Disord Soc 1998;13(Suppl 3):2-23.

48 Gironell A, Kulisevsky J. Diagnosis and management of essential tremor and dystonic tremor. Ther Adv Neurol Disord $2009 ; 2: 215-22$.

49 Cardoso F. Difficult diagnoses in hyperkinetic disorders-a focused review. Front Neurol 2012;3:151.

50 Thanvi B, Lo N, Robinson T. Essential tremor-the most common movement disorder in older people. Age Ageing 2006;35:344-9.

51 Chandran V, Pal PK. Essential tremor: beyond the motor features. Parkinsonism Relat Disord 2012;18:407-13.

52 Berry-Kravis E, Abrams L, Coffey SM, et al. Fragile $\mathrm{X}$-associated tremor/ataxia syndrome: clinical features, genetics, and testing guidelines. Mov Disord Off J Mov Disord Soc 2007;22:2018-30, quiz 2140.

53 Capelli LP, Gonçalves MRR, Leite CC, et al. The fragile $\mathrm{x}$-associated tremor and ataxia syndrome (FXTAS). Arq Neuropsiquiatr 2010;68:791-8.

54 Ling H, Massey LA, Lees AJ, et al. Hypokinesia without decrement distinguishes progressive supranuclear palsy from Parkinson's disease. Brain J Neurol 2012;135(Pt 4):1141-53.

55 Kurata T, Kametaka S, Ohta Y, et al. PSP as distinguished from CBD, MSA-P and PD by clinical and imaging differences at an early stage. Intern Med Tokyo Jpn 2011;50:2775-81.

56 Williams DR, Lees AJ. What features improve the accuracy of the clinical diagnosis of progressive supranuclear palsy-parkinsonism (PSP-P)? Mov Disord Off J Mov Disord Soc 2010;25:357-62.

57 Thanvi B, Lo N, Robinson T. Vascular parkinsonism-an important cause of parkinsonism in older people. Age Ageing 2005;34:114-19.

58 Glass PG, Lees AJ, Bacellar A, et al. The clinical features of pathologically confirmed vascular parkinsonism. J Neurol Neurosurg Psychiatry 2012;83:1027-9.

59 Spaulding SJ, Barber B, Colby M, et al. Cueing and gait improvement among people with Parkinson's disease: a meta-analysis. Arch Phys Med Rehabil 2013;94:562-70.

60 Klassen BT, Ahlskog JE. Normal pressure hydrocephalus: how often does the diagnosis hold water? Neurology 2011;77:1119-25.

61 Kiefer M, Unterberg A. The differential diagnosis and treatment of normal-pressure hydrocephalus. Dtsch Ärztebl Int 2012;109:15-25; quiz 26.

62 Cabral D, Beach TG, Vedders L, et al. Frequency of Alzheimer's disease pathology at autopsy in patients with clinical normal pressure hydrocephalus. Alzheimers Dement J Alzheimers Assoc 2011;7:509-13. 
63 Ubhi K, Low P, Masliah E. Multiple system atrophy: a clinical and neuropathological perspective. Trends Neurosci 2011;34:581-90.

64 Rehman HU. Multiple system atrophy. Postgrad Med J 2001;77:379-82.

65 Ludolph AC, Kassubek J, Landwehrmeyer BG, et al. Tauopathies with parkinsonism: clinical spectrum, neuropathologic basis, biological markers, and treatment options. Eur J Neurol Off J Eur Fed Neurol Soc 2009;16:297-309.

66 Rinne JO, Lee MS, Thompson PD, et al. Corticobasal degeneration. A clinical study of 36 cases. Brain J Neurol 1994;117(Pt 5):1183-96.

67 Wu C-K. Parkinson's disease with dementia, lewy-body disorders and alpha-synuclein: recent advances and a case report. Acta Neurol Taiwanica 2011;20:4-14.

68 McKeith I. Dementia with Lewy bodies and Parkinson's disease dementia: where two worlds collide. Pract Neurol 2007;7:374-82.

69 Sikk K, Haldre S, Aquilonius S-M, et al. Manganese-Induced Parkinsonism due to Ephedrone Abuse. Park Dis 2011;2011:865319.

70 Taylor KSM, Counsell C. Is it Parkinson's disease, and if not, what is it? Pract Neurol 2006;6:154-64.

71 Burkhard PR. Acute and subacute drug-induced movement disorders. Parkinsonism Relat Disord 2014;20(Suppl 1): S108-112.

72 Morris HR. Genetics of Parkinson's disease. Ann Med 2005;37:86-96.

73 Lohmann E, Thobois S, Lesage S, et al. A multidisciplinary study of patients with early-onset PD with and without parkin mutations. Neurology 2009;72:110-16.

74 Gilks WP, Abou-Sleiman PM, Gandhi S, et al. A common LRRK2 mutation in idiopathic Parkinson's disease. Lancet 2005;365:415-16.

75 Zarranz JJ, Alegre J, Gómez-Esteban JC, et al. The new mutation, E46 K, of alpha-synuclein causes Parkinson and Lewy body dementia. Ann Neurol 2004;55:164-73.

76 Kasten M, Klein C. The many faces of alpha-synuclein mutations. Mov Disord Off J Mov Disord Soc 2013;28:697-701.

77 Poulopoulos M, Levy OA, Alcalay RN. The neuropathology of genetic Parkinson's disease. Mov Disord Off J Mov Disord Soc 2012;27:831-42.

78 Kilarski LL, Pearson JP, Newsway V, et al. Systematic review and UK-based study of PARK2 (parkin), PINK1, PARK7 (DJ-1) and LRRK2 in early-onset Parkinson's disease. Mov Disord Off J Mov Disord Soc 2012;27:1522-9.

79 Alcalay RN, Caccappolo E, Mejia-Santana H, et al. Cognitive and motor function in long-duration PARKIN-associated Parkinson disease. JAMA Neurol 2014;71:62-7.

80 Wickremaratchi MM, Knipe MDW, Sastry BSD, et al. The motor phenotype of Parkinson's disease in relation to age at onset. Mov Disord Off J Mov Disord Soc 2011;26:457-63.

81 Walshe JM, Yealland M. Wilson's disease: the problem of delayed diagnosis. J Neurol Neurosurg Psychiatry 1992;55:692-6.

82 Lorincz MT. Neurologic Wilson's disease. Ann N Y Acad Sci 2010;1184:173-87.

83 Roos RAC. Huntington's disease: a clinical review. Orphanet J Rare Dis 2010;5:40.
84 Teive HAG. Spinocerebellar ataxias. Arq Neuropsiquiatr 2009;67:1133-42.

85 Espay AJ, Litvan I. Parkinsonism and frontotemporal dementia: the clinical overlap. J Mol Neurosci 2011;45:343-9.

86 Boeve BF, Hutton M. Refining frontotemporal dementia with parkinsonism linked to chromosome 17: introducing FTDP-17 (MAPT) and FTDP-17 (PGRN). Arch Neurol 2008;65:460-4.

87 Liu Y, Yu J-T, Sun F-R, et al. The clinical and pathological phenotypes of frontotemporal dementia with C9ORF72 mutations. J Neurol Sci 2013;335:26-35.

88 Batista-Nascimento L, Pimentel C, Menezes RA, et al. Iron and neurodegeneration: from cellular homeostasis to disease. Oxid Med Cell Longev 2012;2012:128647.

89 Gregory A, Polster BJ, Hayflick SJ. Clinical and genetic delineation of neurodegeneration with brain iron accumulation. J Med Genet 2008;46:73-80.

90 Gregory A, Hayflick SJ. Neurodegeneration with brain iron accumulation. Folia Neuropathol Assoc Pol Neuropathol Med Res Cent Pol Acad Sci 2005;43:286-96.

91 Schneider SA, Bhatia KP. Excess iron harms the brain: the syndromes of neurodegeneration with brain iron accumulation (NBIA). J Neural Transm 2013;120:695-703.

92 Schneider SA, Bhatia KP. Preface. Int Rev Neurobiol 2013;110:xv-xviii.

93 NICE. CG35 Parkinson's disease: full guideline [Internet]. NICE. [cited 4 Apr 2014]. http://www.nice.org.uk/

94 Suchowersky O, Reich S, Perlmutter J, et al. Practice Parameter: diagnosis and prognosis of new onset Parkinson disease (an evidence-based review): report of the Quality Standards Subcommittee of the American Academy of Neurology. Neurology 2006;66:968-75.

95 Paviour DC, Price SL, Stevens JM, et al. Quantitative MRI measurement of superior cerebellar peduncle in progressive supranuclear palsy. Neurology 2005;64:675-9.

96 Schrag A, Good CD, Miszkiel K, et al. Differentiation of atypical parkinsonian syndromes with routine MRI. Neurology 2000;54:697-702.

97 Benamer HT, Patterson J, Wyper DJ, et al. Correlation of Parkinson's disease severity and duration with 123I-FP-CIT SPECT striatal uptake. Mov Disord Off J Mov Disord Soc 2000;15:692-8.

98 Bajaj N, Hauser RA, Grachev ID. Clinical utility of dopamine transporter single photon emission CT (DaT-SPECT) with (123I) ioflupane in diagnosis of parkinsonian syndromes. J Neurol Neurosurg Psychiatry 2013;84:1288-95.

99 Brooks DJ. Parkinson's disease: diagnosis. Parkinsonism Relat Disord 2012;18(Suppl 1):S31-33.

100 Orimo S, Suzuki M, Inaba A, et al. 123I-MIBG myocardial scintigraphy for differentiating Parkinson's disease from other neurodegenerative parkinsonism: a systematic review and meta-analysis. Parkinsonism Relat Disord 2012;18:494-500.

101 Blazejewska AI, Schwarz ST, Pitiot A, et al. Visualization of nigrosome 1 and its loss in PD: pathoanatomical correlation and in vivo 7 T MRI. Neurology 2013;81:534-40.

102 Piccini P, Brooks DJ. New developments of brain imaging for Parkinson's disease and related disorders. Mov Disord Off J Mov Disord Soc 2006;21:2035-41.

103 Kajimoto Y, Miwa H, Kondo T. Transcranial sonography in relation to SPECT and MIBG. Int Rev Neurobiol 2010;90:48-62. 